Supporting Information for

\title{
Conformational Scaling Relations of Two-Dimensional Macromolecular Graphene Oxide in Solution
}

Peng $\mathrm{Li}^{\dagger}$, Shijun Wang ${ }^{\ddagger}$ Fanxu Meng ${ }^{\dagger}$, Ya Wang ${ }^{\dagger}$, Fan Guo ${ }^{\dagger}$, Sangeetha Rajendran ${ }^{\dagger}$, Chao Gao ${ }^{\dagger}$, Zhiping $\mathrm{Xu}^{* \star}$, Zhen $\mathrm{Xu}^{* \dagger}$

$\dagger$ MOE Key Laboratory of Macromolecular Synthesis and Functionalization, Department of Polymer Science and Engineering, Key Laboratory of Adsorption and Separation Materials \& Technologies of Zhejiang Province, Zhejiang University, 38 Zheda Road, Hangzhou 310027, P. R. China

$\$$ Applied Mechanics Laboratory, Department of Engineering Mechanics and Center for Nano and Micro Mechanics, Tsinghua University, Beijing 100084, P. R. China

Table of Contents
A. Experimental Details
B. Simulation Details
C. Viscoelastic scaling relations of GO solutions
D. Supplemental Tables, Figures and Captions
E. Supplemental References 


\section{A. Experimental Details}

Materials. Graphite crystals with different sizes were purchased from Qingdao Henglide Graphite Co., Ltd. Concentrated $\mathrm{H}_{2} \mathrm{SO}_{4}(98 \%), \mathrm{KMnO}_{4}, \mathrm{H}_{2} \mathrm{O}_{2}(30 \%)$, ethyl acetate (EA), acetone and dimethylformamide (DMF) were purchased from Sinopharm Chemical Reagent Co. Ltd. and used as received.

Instruments. Scanning electron microscopy (SEM) images were taken on a Hitachi S4800 field-emission SEM system. Atomic force microscopy (AFM) images of single-layer graphene oxide (GO) sheets were taken in the tapping mode on an NSK SPI3800 with samples prepared by spin-coating diluted solutions onto freshly exfoliated mica substrates at 1000 r.p.m. X-ray photoelectron spectroscopy (XPS) was performed using a PHI 5000C ESCA system operated at $14.0 \mathrm{kV}$ with the $\mathrm{C} 1 \mathrm{~s}$ neutral carbon peak at $284.8 \mathrm{eV}$ used as reference binding energies. Static light scattering (SLS) was carried out using ALG/CGS3. Cryo-TEM inspection was taken on Titan Krios 200kV TEM. The liquid specimens were dropped onto the copper grid and then quickly frozen in liquid ethane at $-183^{\circ} \mathrm{C}$ to assure that GO was embedded in a thin layer of amorphous ice. 


\section{B. Simulation Details}

Dissipative particle dynamics (DPD) models for solvents and GO. DPD simulations are implemented to explore the dynamic behaviors of dilute GO solution, where both the GO sheets and solvent are represented using coarse-grained beads. The equations of motion for all the solvent beads and non-bonded GO beads (but not for the bonded GO beads that are modelled using a coarse-grained force field including bond-stretch and dihedral terms) are [1]

$\mathbf{F}_{i}=\sum_{j \neq i}\left(\mathbf{F}_{i j} \mathrm{C}+\mathbf{F}_{i j} \mathrm{D}+\mathbf{F}_{i j}^{\mathrm{R}}\right)$

where the superscripts $\mathrm{C}, \mathrm{D}$, and $\mathrm{R}$ denote conservative, dissipative, and random forces, respectively, and

$\mathbf{F}_{i j} \mathrm{C}=a_{i j}\left(1-r_{i j} / R_{\mathrm{c}}\right) \hat{\mathbf{r}}_{i j}, r_{i j}<R_{\mathrm{c}}$

$\mathbf{F}_{i j} \mathrm{D}=-b w^{\mathrm{D}}\left(r_{i j}\right)\left(\hat{\mathbf{r}_{i j}} \cdot \mathbf{v}_{i j}\right) \hat{\mathbf{r}_{i j}}$

$\mathbf{F}_{i j}^{\mathrm{R}}=\sigma_{\mathrm{R}} w^{\mathrm{R}}\left(r_{i j}\right) \theta_{i j} \hat{\mathbf{r}_{i j}}$

where $\mathbf{v}_{i j}=\mathbf{v}_{i}-\mathbf{v}_{j}, \mathbf{r}_{i j}=\mathbf{r}_{i}-\mathbf{r}_{j}, \hat{\mathbf{r}_{i j}}=\mathbf{r}_{i j} / r_{i j}, a_{i j}$ is the conservative force coefficient for a pair of beads of type $i$ and $j$, and $R_{\mathrm{c}}$ is the cut-off distance for this soft repulsion. The coefficients $b$ (in term $F_{i j} \mathrm{D}$ ) and $\sigma_{\mathrm{R}}$ (in term $F_{i j}^{\mathrm{R}}$ ) define the amplitudes of the dissipative and random forces, respectively. Here the weight functions $w^{\mathrm{D}}, w^{\mathrm{R}}$ vanish at $r_{i j}>R_{\mathrm{c}}, \theta_{i j}$ is a Gaussian white noise satisfying the conditions that $\left\langle\theta_{i j}(t)>=0\right.$ and $\left\langle\theta_{i j}(t) \theta_{k l}\left(t^{\prime}\right)\right\rangle=\left(\delta_{i k} \delta_{j l}+\delta_{i l} \delta_{j k}\right) \delta(t$ $-t^{\prime}$ ), and the amplitude of noise $\sigma_{\mathrm{R}}$ is fixed as 3 following the work by Groot et al. [1]. To ensure that the Boltzmann distribution is achieved at thermal equilibrium, we enforce $w^{\mathrm{D}}\left(r_{i j}\right)=\left[w^{\mathrm{R}}\left(r_{i j}\right)\right]^{2}$ and $\sigma 2 \mathrm{R}=2 b k_{\mathrm{B}} T$. For simplicity, $w^{\mathrm{D}}\left(r_{i j}\right)$ is chosen to be $1-r_{i j} / R_{\mathrm{c}}$, which defines the same interaction region as that for the conservative force.

We consider water solvent where each water molecule takes up $\sim 30 \AA^{3}$. Three of them are grouped into one DPD bead, that is, the number of water molecules per bead is $N_{\mathrm{m}}=3$. One bead thus occupies a volume of $V_{\mathrm{w}}=90 \AA^{3}$, and the number density $\rho$ of DPD beads, defined as the number of the solvent beads per cubic volume $R_{\mathrm{c}}{ }^{3}$, is 3 . This determines the mass $m$ and cut-off distance $R_{\mathrm{c}}$ for the beads. The mass $m$ of solvent beads, temperature $T$, 
and cut-off distance $R_{\mathrm{c}}$ are defined in the unit of mass, energy, and length, respectively. The unit of time is $\tau=R_{\mathrm{c}}\left(m / k_{\mathrm{B}} T\right)^{0.5}$, and the time step of simulation is $\delta t=0.01 \tau$. The physical value of the time scale $\tau$ is determined by $\tau=N_{\mathrm{m}} D_{\mathrm{DPD}} R_{\mathrm{c}}{ }^{2} / D_{\text {water }}$, where $D_{\mathrm{DPD}}$ and $D_{\text {water }}$ are the diffusion constants for DPD beads and water molecules, respectively [2]. Expressed in DPD units, we have $m=k_{\mathrm{B}} T=R_{\mathrm{c}}=\tau=1$. The conversion between values in the DPD and physical units are listed in Table S1.

The coarse-grained model for GO sheets is constructed based on a hexagonal lattice as shown in Figure 1b. The edges are modelled as linear-elastic springs and a pair of triangular facets sharing a common edge is modelled as a dihedral. Atoms in a GO sheet are grouped into beads with equal masses. Bonding interaction between beads is modelled through a stretching energy term $E_{\mathrm{s}}=k_{\mathrm{s}}\left(r-r_{0}\right)^{2} / 2$ with a stiffness $k_{\mathrm{s}}=\frac{\sqrt{3}}{2} Y t$, where $r_{0}$ is the equilibrium inter-bead distance, and $Y t$, the product of Young's modulus and thickness, is the 2D tensile stiffness of the GO sheet [3]. The bending resistance of a GO sheet is modelled by a dihedral term $E_{\mathrm{b}}=k_{\mathrm{d}}\left(1-\mathbf{n}_{i} \mathbf{n}_{j}\right)$ with a stiffness $k_{\mathrm{d}}=\frac{2}{\sqrt{3}} D$, where $\mathbf{n}_{i}, \mathbf{n}_{j}$ are the unit normal vectors of triangles $i$ and $j$ sharing a common edge and $D$ is the bending stiffness of GO. This dihedral term is equivalently expressed using a harmonic potential of dihedral angle $\varphi$ as $E_{\mathrm{b}}=k_{\mathrm{d}}(1+\cos \varphi)$. Here we use $D$ instead of $Y I$ to avoid the ambiguity in the definition of the thickness $t$ for of single-atom-thick sheets. The bending stiffness of GO is reported to be $\sim 1 k_{\mathrm{B}} T$ in room temperature [4], and thus the bending stiffness our models is chosen within $0 \sim 10 k_{\mathrm{B}} T$. The conservative force coefficients $a_{i j}$ between nonbonding GO beads (solvent and GO) are set as the same as those between solvent beads, $a_{i j}=78[2]$.

The implementation of adhesion between GO membranes follows previous studies [5, 6], where pairwise interaction between non-bonding beads (beads not interacting through the bond-stretch and dihedral terms) in GO sheets is added to the DPD force field, in the form of Lennard-Jones 12-6 potential $E_{\mathrm{L}-\mathrm{J}}=4 \varepsilon\left[(\sigma / r)^{12}-(\sigma / r)^{6}\right]$ with a cut-off distance of 
$2.5 \sigma$. Here we set $2^{1 / 6} \sigma=R_{\mathrm{c}}$. The adhesion energy density between two GO sheets (the reduction in energy by forming a face-to-face contact divided by the surface area, or twice the area of a GO sheet) is calculated from a specific value of the strength of inter-bead interaction, $\varepsilon$. Fitting to the value $\Gamma_{0}=0.31 \mathrm{~J} / \mathrm{m}^{2}$ experimentally measured for graphene yields $\varepsilon_{0}=4.41 k_{\mathrm{B}} T$ per pair of beads [7]. To consider the solvent effects, we have $\varepsilon / \varepsilon_{0}<1$ for the presence of electrostatic repulsion [8]. The coarse-grained length scale or the interbead distance in simulations ( $0.452 \mathrm{~nm}$ for GO beads, $0.646 \mathrm{~nm}$ for solvent beads) is much shorter than the length scale of contact between GO sheets and thus the adhesion behaviors can be well captured.

DPD simulation setup. The DPD simulations are performed by using the large-scale atomic/molecular massively parallel simulator (LAMMPS) [9]. The size of the supercell is $30 R_{\mathrm{c}}$ containing $\sim 81,000 \mathrm{DPD}$ beads and the lateral length of the GO sheets is set to range from $7 R_{\mathrm{c}}$ to $35 R_{\mathrm{c}}$. The $\mathrm{GO}$ solutions are relaxed over $2.0 \times 10^{6}$ timesteps with $k_{\mathrm{B}} T=1$, corresponding to $300 \mathrm{~K}$. After equilibration, the shear viscosity is calculated using the Green-Kubo formalism through the auto-correlation function of pressure tensor (PACF) $\mathbf{P}$ [10]

$\eta=\frac{V}{3 k_{\mathrm{B}} T} \int_{0}^{\infty}<P_{i j}(t) P_{i j}(0)>\mathrm{d} t$

where $V$ is the volume of the supercell, $P_{i j}(i \neq j)$ are the components of pressure tensor, and $t$ is the time measured from the starting point in the equilibrium simulation. The PACF is calculated over 40,000 samples of bead trajectories, and each of them is integrated for 4,000 timesteps. The intrinsic viscosity of GO is defined as

$[\eta]=\lim _{c \rightarrow 0} \frac{\eta-\eta_{0}}{c \eta_{0}}=\lim _{c \rightarrow 0} f(c)$

where $\eta_{0}$ is the viscosity measured at a concentration of $c=0$.

The radius of gyration tensor, $\mathbf{S}$, is defined as 
$\mathbf{S}=\frac{1}{N} \sum_{i}\left[\begin{array}{ccc}\left(\mathrm{x}_{i}-x_{\mathrm{cm}}\right)^{2} & \left(x_{i}-x_{\mathrm{cm}}\right)\left(y_{i}-y_{\mathrm{cm}}\right) & \left(x_{i}-x_{\mathrm{cm}}\right)\left(z_{i}-z_{\mathrm{cm}}\right) \\ \left(y_{i}-y_{\mathrm{cm}}\right)\left(x_{i}-x_{\mathrm{cm}}\right) & \left(y_{i}-y_{\mathrm{cm}}\right)^{2} & \left(y_{i}-y_{\mathrm{cm}}\right)\left(z_{i}-z_{\mathrm{cm}}\right) \\ \left(z_{i}-z_{\mathrm{cm}}\right)\left(x_{i}-x_{\mathrm{cm}}\right) & \left(z_{i}-z_{\mathrm{cm}}\right)\left(y_{i}-y_{\mathrm{cm}}\right) & \left(z_{i}-z_{\mathrm{cm}}\right)^{2}\end{array}\right]$

where $N$ is the number of beads, $\left(x_{i}, y_{i}, z_{i}\right)$ is the coordinates of the $i$-th bead, and $\left(x_{\mathrm{cm}}, y_{\mathrm{cm}}\right.$, $\left.z_{\mathrm{cm}}\right)$ is the center-of-mass coordinates. The eigenvalues of $\mathbf{S}$ are denoted as $\lambda_{1}>\lambda_{2}>\lambda_{3}$ and the radius of gyration is $R_{\mathrm{g}}=\left(\lambda_{1}+\lambda_{2}+\lambda_{3}\right)^{1 / 2}$. The relative shape anisotropy can be computed from the eigenvalues of $\mathbf{S}$ as [11]

$\kappa^{2}=1-3 \frac{\lambda_{1} \lambda_{2}+\lambda_{2} \lambda_{3}+\lambda_{3} \lambda_{1}}{\left(\lambda_{1}+\lambda_{2}+\lambda_{3}\right)^{2}}$

which reflects both the symmetry and dimensionality of a macromolecule, also known as the shape descriptor, and has a value between 0 (spheres) and 1 (rods).

The solvent accessible surface area (SASA) [12] is calculated by considering a radius of the beads as $1 R_{\mathrm{c}}=6.46 \AA$, the calculation of which is implemented in the visual molecular dynamics (VMD) [13].

The hydrodynamic radius, $R_{\mathrm{h}}$, is computed using Stokes-Einstein relations,

$D_{\mathrm{t}}=\frac{k_{\mathrm{B}} T}{6 \pi \eta_{0} R_{\mathrm{h}}}$

$D_{\mathrm{r}}=\frac{k_{\mathrm{B}} T}{8 \pi \eta_{0} R_{\mathrm{h}}^{3}}$

where $\eta_{0}$ is the viscosity calculated for the solvent. $D_{\mathrm{t}}$ and $D_{\mathrm{r}}$ are the translational and rotational diffusion coefficients that are calculated by the mean square displacement (MSD) and angular mean square displacement (AMSD), respectively $[14,15]$. To calculate the AMSD, we track the rotational displacement of the eigenvectors of $\mathbf{S}$. 


\section{Viscoelastic scaling relations of GO solutions}

We performed DPD simulations to calculate the storage $\left(G^{\prime}\right)$ and loss modulus $\left(G^{\prime \prime}\right)$ as functions of $L$ (Figure S11). The shear viscosity can be evaluated from the moduli as $\eta=$ $\left[\left(G^{\prime}\right)^{2}+\left(G^{\prime \prime}\right)^{2}\right]^{1 / 2} / \omega[16]$. In the simulations, a sinusoidal form of shear strain is applied to the simulation box with an amplitude of 0.1 and a period of $40 \tau$. We find that $G^{\prime}<G^{\prime \prime}$, which suggests that the viscous effect is dominant in the dilute GO solution modelled here. Conformational (flat and crumpled) dependence is identified. $G^{\prime \prime}$ increases with $L$ for nearly-flat GO, but decreases for the crumpled one. This could be explained by the fact that the hydrodynamic radius $R_{\mathrm{h}}$ scales as $L$ for flat GO but $\sim L^{\gamma}$ for crumpled ones $(\gamma \sim$ 2/3), and the interaction between GO and solvents increases with $R_{\mathrm{h}}$ (Figures $4 \mathbf{a}$ and $\mathbf{6}$ ).

The simulation results summarized in Figure S11 were fitted into scaling relations between the moduli and GO size. The exponent $v$ depends on the concentration and conformations of GO. At the same concentration, GO with the flat conformation has larger $v$ values than that with the crumpled conformation for both $G^{\prime}$ and $G^{\prime \prime}$.

The viscoelastic behaviors of solutions are usually concerned at a high concentration, and the behaviors may depend on the amplitude and frequency of loads and concentration. Experimental studies suggest that the modulus and yield stress of GO solution (at a concentration (20 mg/mL) much higher than our dilute solution) increase with the size $L$ $[17,18]$.

This work is focused on the dilute solution of GO, and the discussion is thus limited to the intrinsic viscosity and critical overlapping concentration. 


\section{Supplemental Tables, Figures, and Captions}

Table S1. The table of conversion for DPD and physical units.

\begin{tabular}{ll}
\hline DPD units & Conversion relations \\
\hline Cut-off distance, $R_{\mathrm{c}}$ & $1 R_{\mathrm{c}}=6.46 \AA$ \\
Mass of beads, $m$ & $1 \mathrm{~m}=54 \mathrm{~g} / \mathrm{mol}$ \\
Simulation temperature, $T$ & $1 k_{\mathrm{B}} T=0.59 \mathrm{kcal} / \mathrm{mol}$ \\
Simulation time scale, $\tau$ & $1 \tau=88 \mathrm{ps}$ \\
\hline
\end{tabular}


Table S2. Parameters for the GO sheets used in the DPD simulations.

\begin{tabular}{lll}
\hline Parameters & Units & Values \\
\hline Equilibrium inter-bead distance, $r_{0}$ & $R_{\mathrm{c}}$ & 0.7 \\
Tensile stiffness, $k_{\mathrm{s}}$ & $k_{\mathrm{B}} T / \AA^{2}$ & 200 \\
Equilibrium angle, $\varphi_{0}$ & degree & 180 \\
Dihedral bending stiffness, $k_{\mathrm{d}}$ & & $0 \sim 10$ \\
\hline
\end{tabular}


(a)
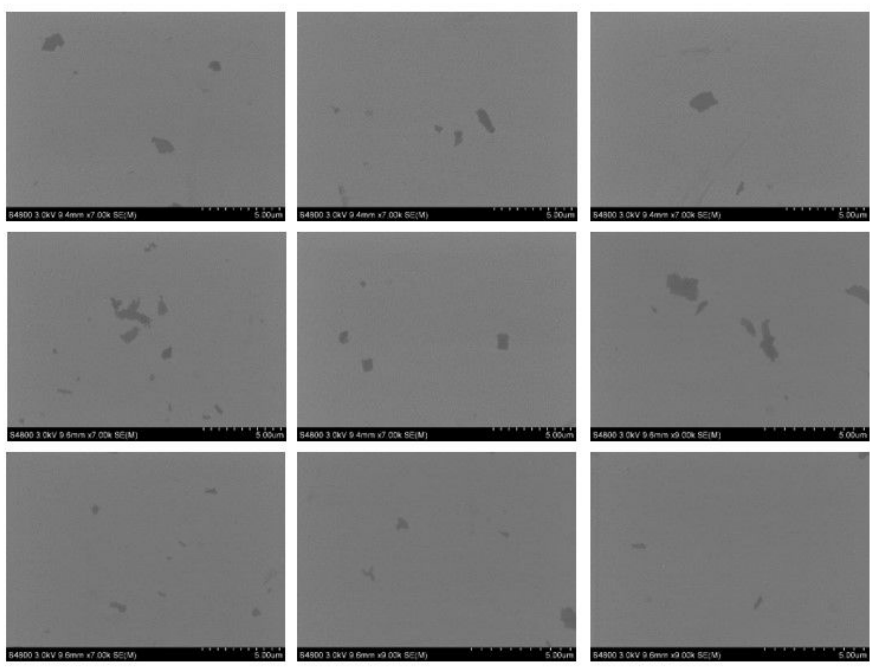

(d) (c)
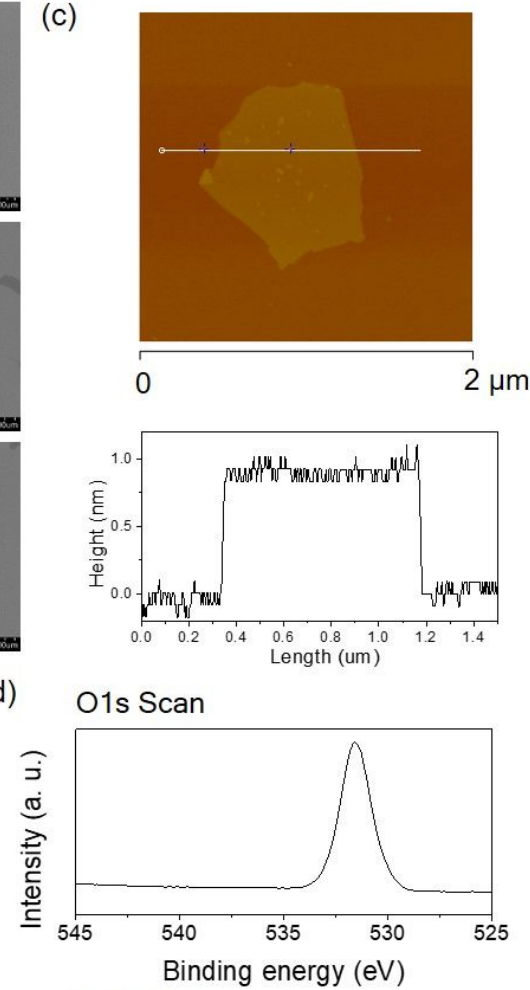

(b)

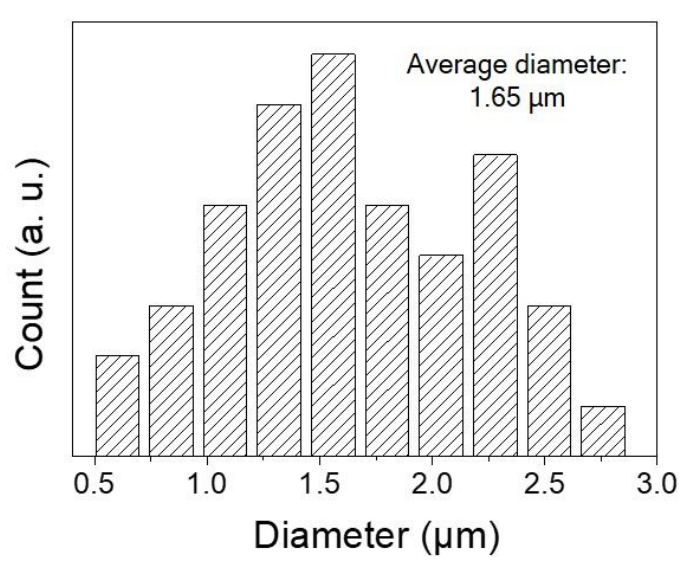

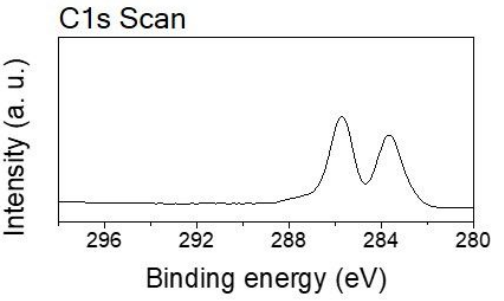

Figure S1. SEM images (a), statistic distribution of GO sizes (b), the AFM image (c) of deposited samples on the silica substrate and XPS spectra (d) of GO with an average size of $1.65 \mu \mathrm{m}$. 
(a)
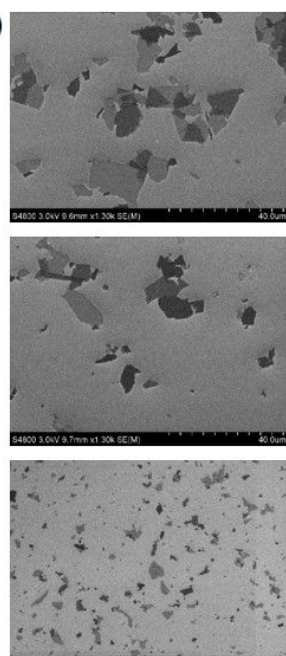

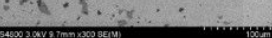
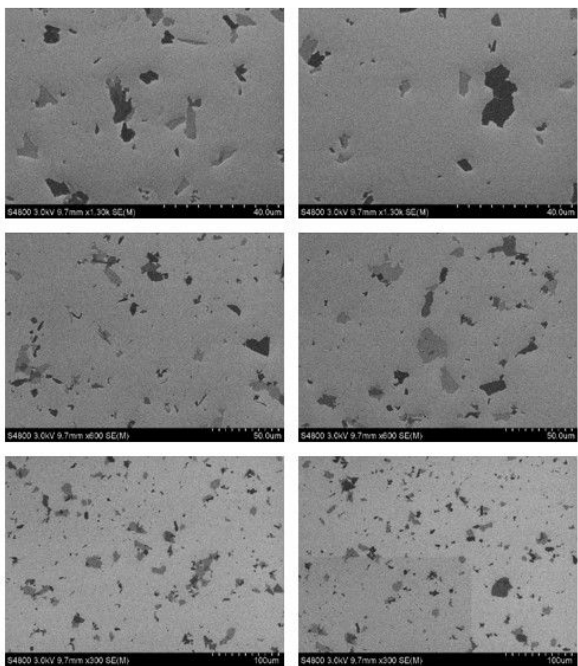

(c)
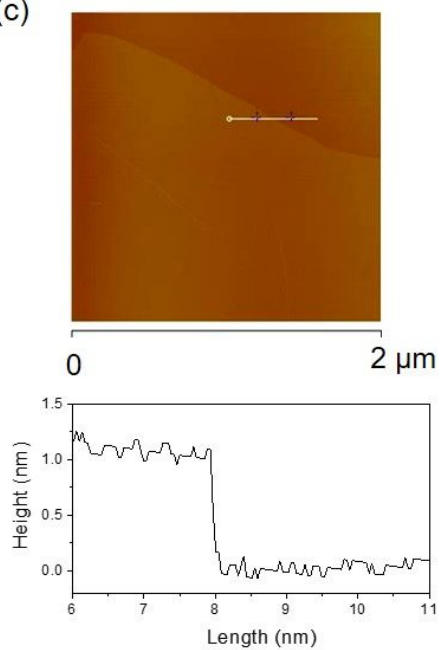

(b)

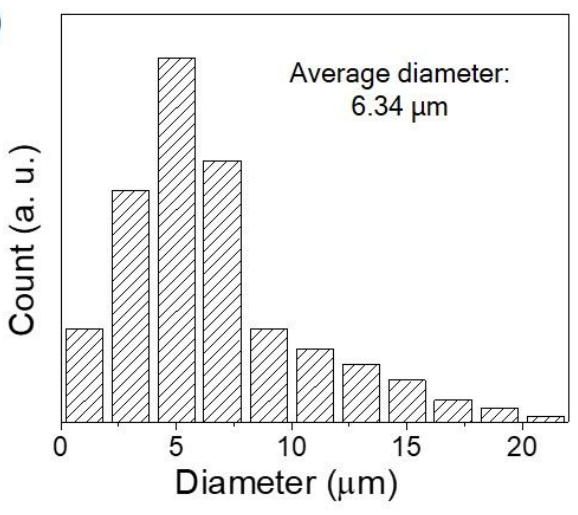

(d)
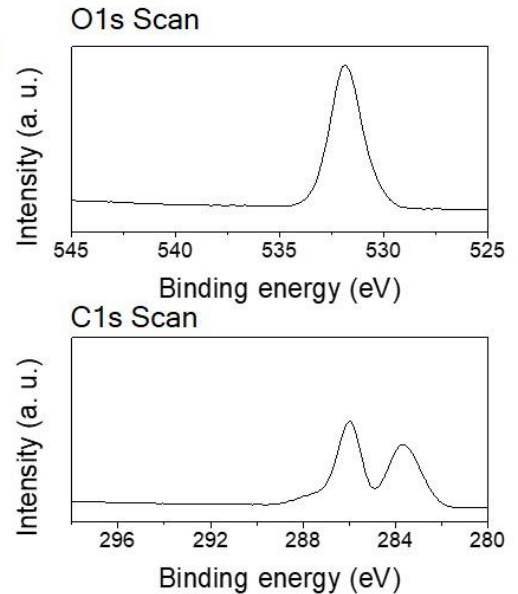

Figure S2. SEM images (a), size distribution (b), the AFM image (c) of a deposited sample on the silica substrate and XPS spectra (d) of GO with an average size of $6.34 \mu \mathrm{m}$. 
(a)
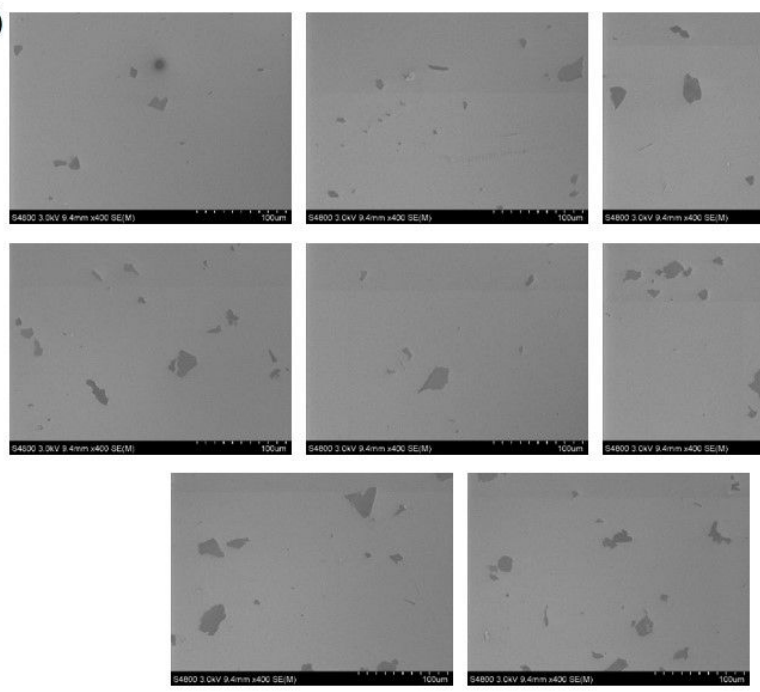

(b)

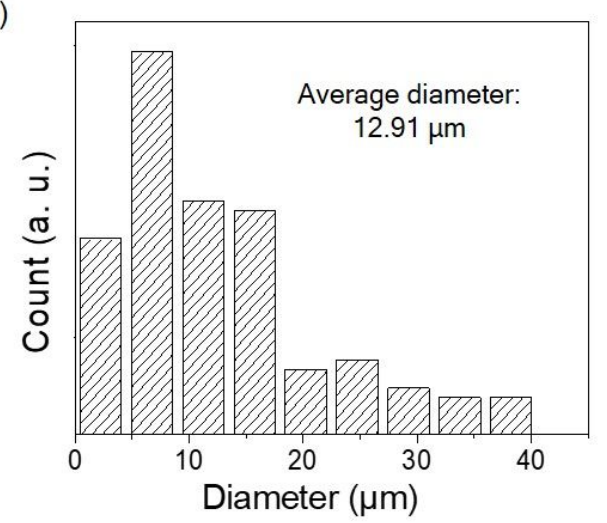

(c)
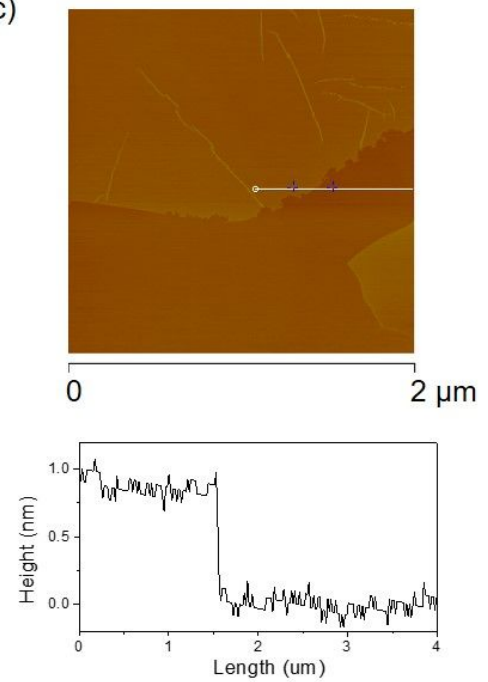

(d)
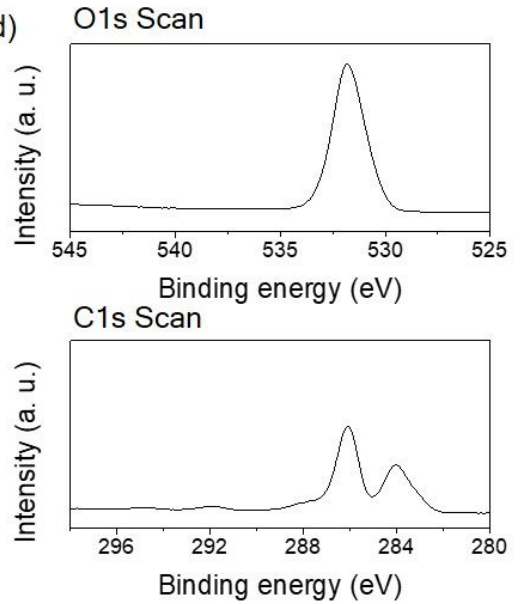

Figure S3. SEM images (a), size distribution (b), the AFM image (c) of a deposited sample on the silica substrate and XPS spectra (d) of GO with an average size of $12.91 \mu \mathrm{m}$. 
(a)
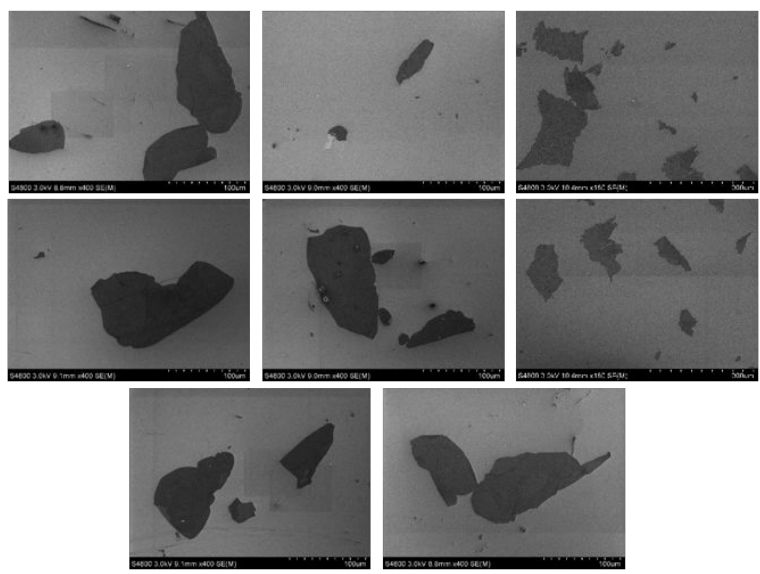

(c) 01s Scan

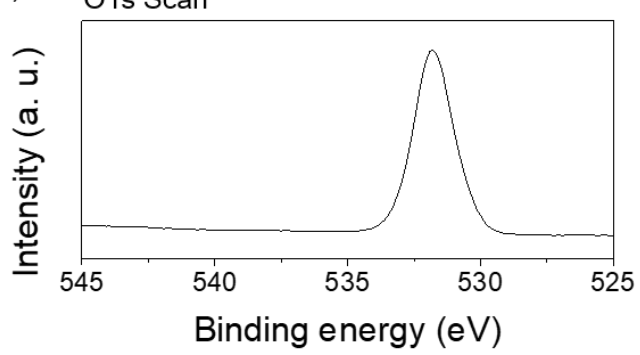

(b)

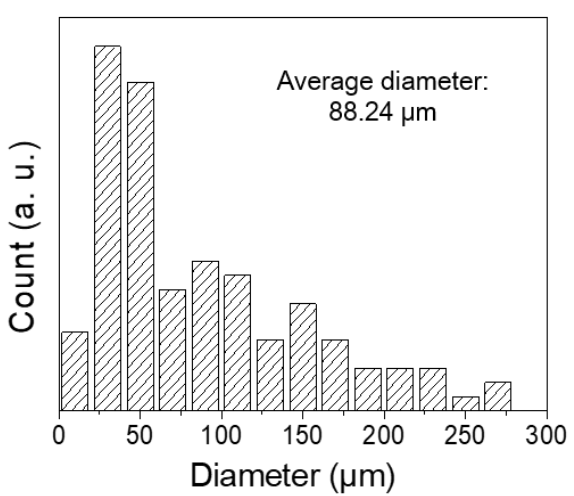

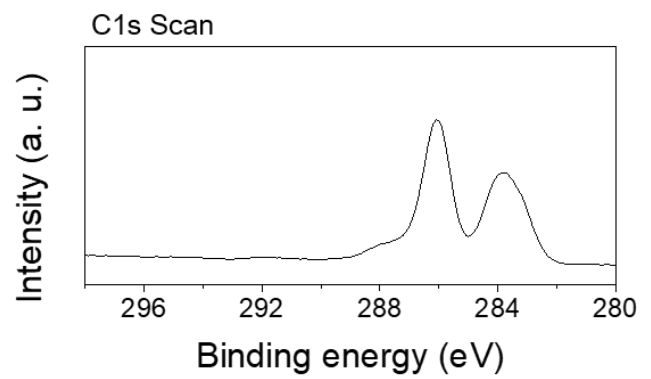

Figure S4. SEM images (a), statistic distribution of sizes (b), XPS spectra (c) of GO with an average size of $88.24 \mu \mathrm{m}$. 
(a)
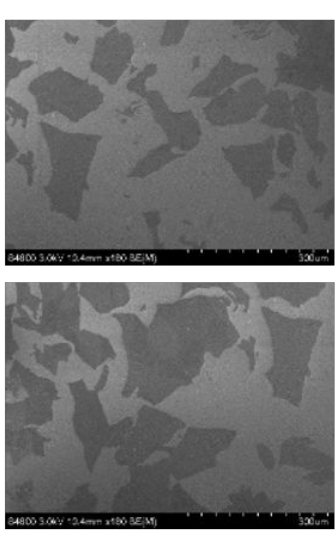

(c) 01s Scan
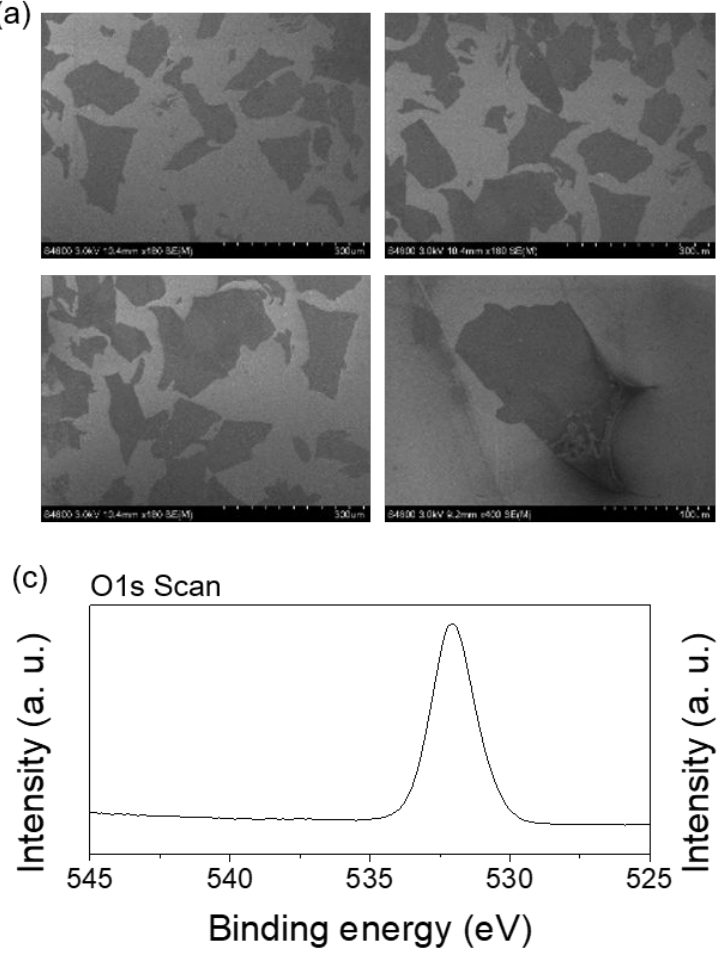

(b)

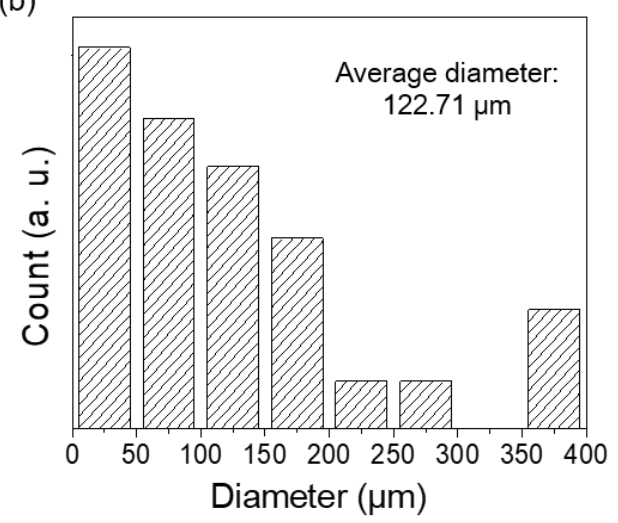

C1s Scan

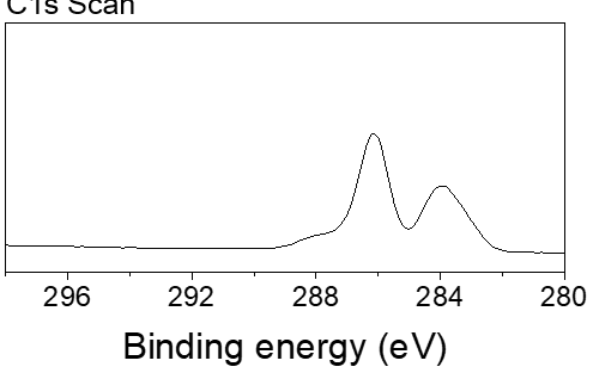

Figure S5. SEM images (a), statistic distribution of sizes (b), XPS spectra (c) of GO with an average size of $122.71 \mu \mathrm{m}$. 

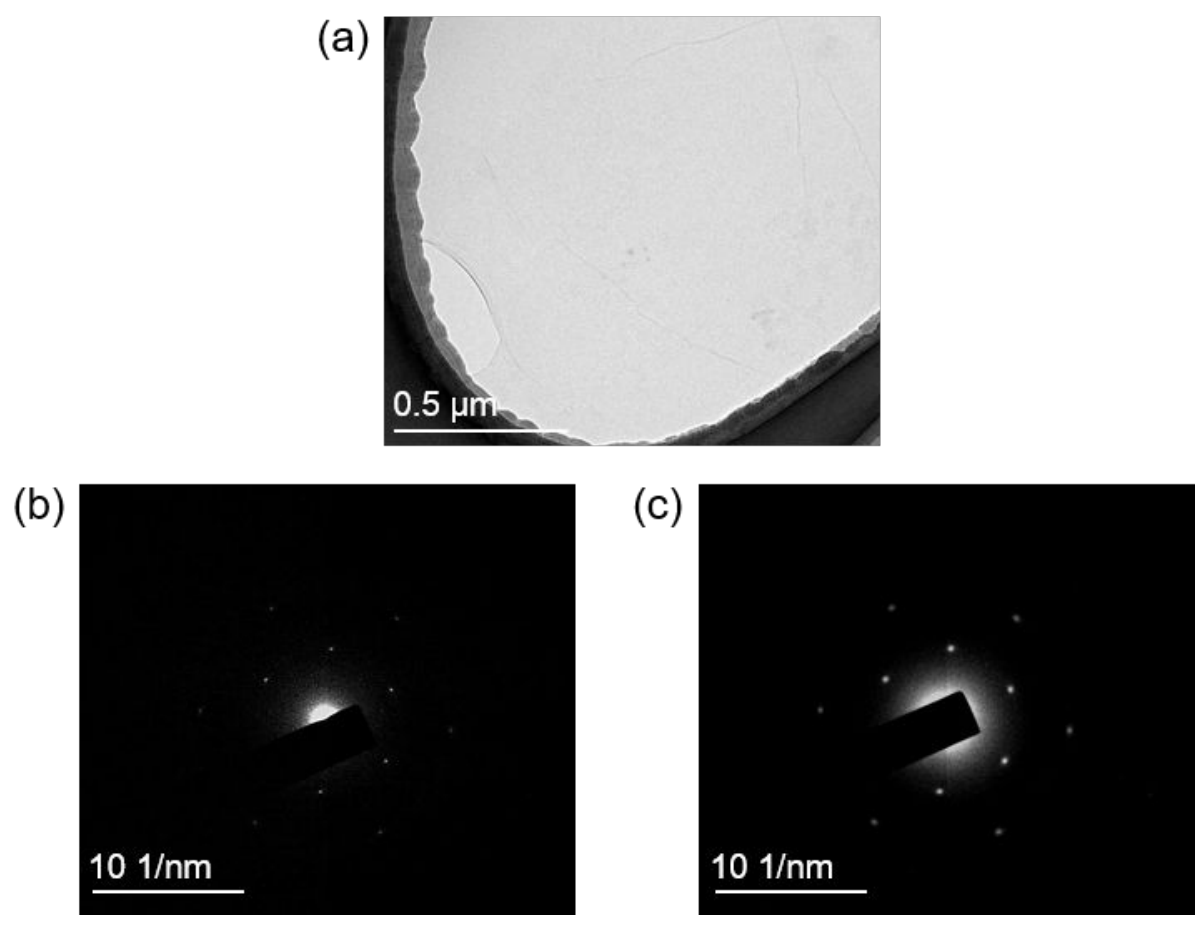

Figure S6. (a) TEM images of single-layer GO sheets deposited onto a copper grid. (b, c) Selected area electron diffraction (SAED) images that demonstrate partially-preserved crystallinity of $s p^{2}$ graphitic lattice after oxidation and exfoliation. 

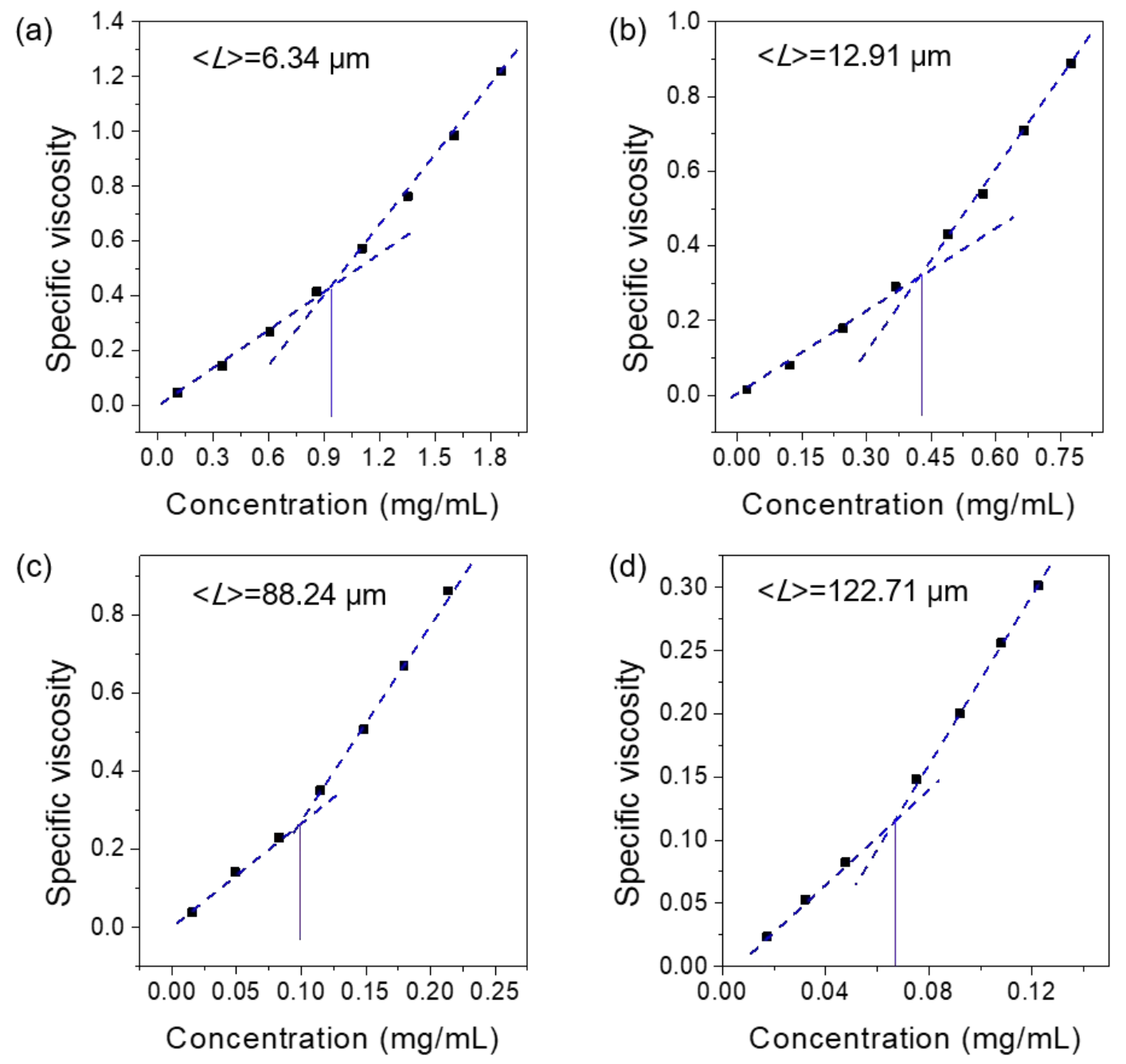

Figure S7. Specific viscosities plotted as a function of the concentration of GO sheets with four different average lateral sizes. The vertical lines denote the overlapping transition. 


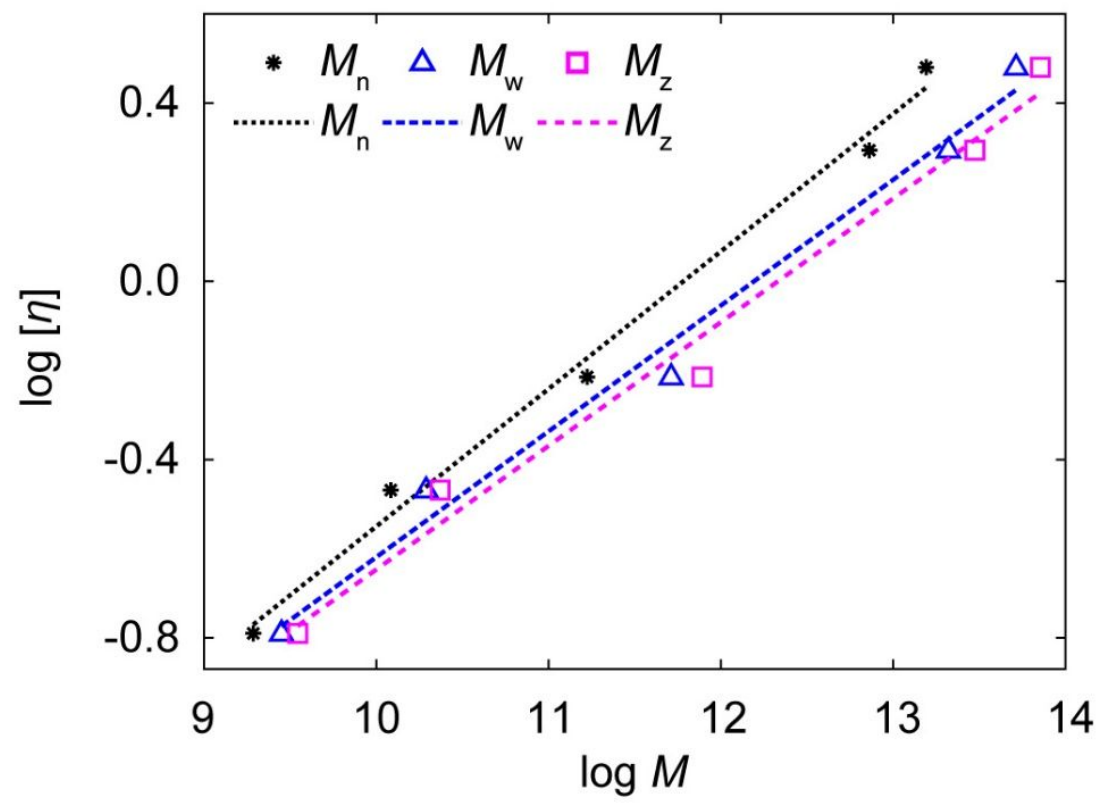

Figure S8. The intrinsic viscosity $[\eta]$ with molecular weights $\left(M_{\mathrm{n}}\right.$, number average; $M_{\mathrm{w}}$, weight average; and $M_{\mathrm{z}}$, Z-average) for GO in DMF. The corresponding MHS relations are deduced as $[\eta] \propto M_{\mathrm{n}}^{0.33},[\eta] \propto M_{\mathrm{w}}^{0.282}$ and $[\eta] \propto M_{\mathrm{z}}^{0.277}$.

Molecular weights are calculated as $M_{\mathrm{n}}=\frac{\sum\left(n_{i} M_{i}\right)}{\sum n_{i}}, M_{\mathrm{w}}=\frac{\sum\left(n_{i} M_{i}^{2}\right)}{\sum\left(n_{i} w_{i}\right)}$ and $M_{\mathrm{z}}=\frac{\sum\left(n_{i} M_{i}^{3}\right)}{\sum\left(n_{i} w_{i}^{2}\right)} . M_{i}$ of each GO sheet is calculated by

$$
M_{i}=\frac{\left(d_{i} / 2\right)^{2} \pi}{S_{0}} \times\left(R \times n_{\mathrm{C}} \times m_{0}+n_{\mathrm{C}} \times m_{\mathrm{C}}\right)
$$

$d_{i}$ : diameter for the $i$-th GO sheet, $S_{0}$ : area of one hexagon carbon ring in graphene, $m_{0}$ : relative atomic mass for oxygen, $m_{\mathrm{C}}$ : relative atomic mass for carbon, $n_{\mathrm{C}}$ : number of carbon atoms in one hexagon ring of graphene, $R$ : ratio of oxygen atoms to carbon atoms obtained from XPS. 


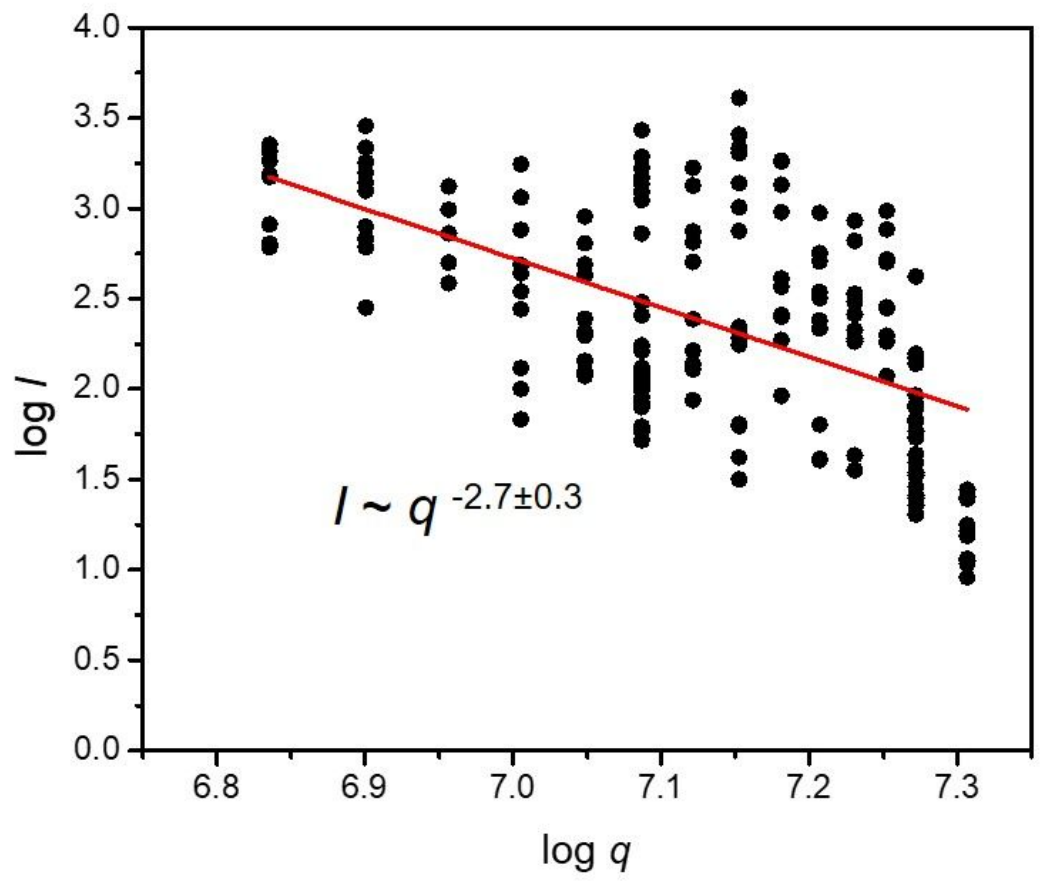

Figure S9. Static light scattering (SLS) results for GO with average lateral size $<L>$ of $122.71 \mu \mathrm{m}$. The scattering intensity $I$ plotted as a function of the scattering vector $q$ (in logarithmic units), exhibiting fluctuation due to variation in the density distribution of large GO sheets in solution, which results in an unacceptable error to the assessment of GO conformation. 


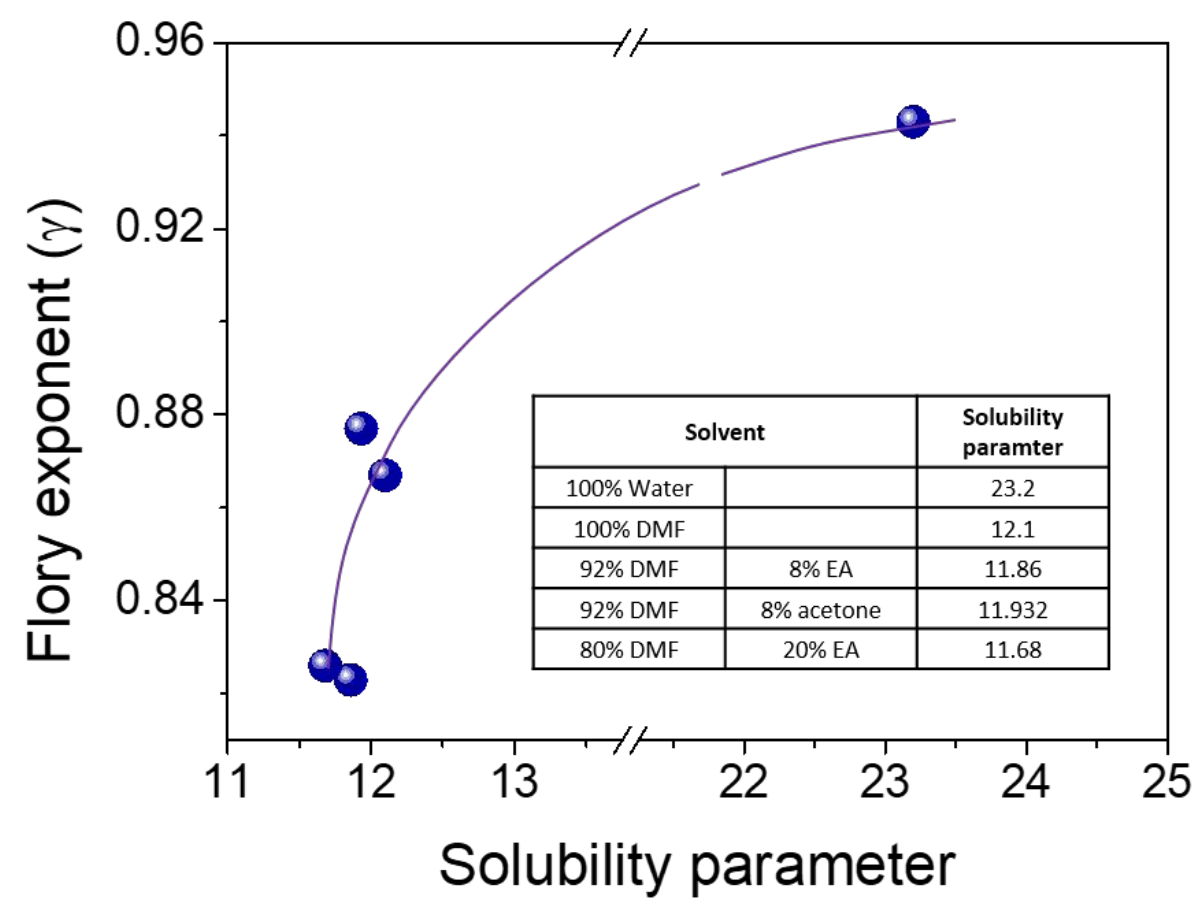

Figure S10. The measured Flory exponent $(\gamma)$ from data in Figure $7 \mathrm{a}$, plotted as a function of the solubility parameter $(\delta)$ of the solvent. The solubility parameters for pure water, DMF, EA, and acetone are taken from Ref. $[19,20]$, and the data for the mixture of the DMF and EA or acetone is estimated as $\delta=\phi_{1} \delta_{1}+\phi_{2} \delta_{2}$, where $\phi_{1}, \phi_{2}$ and $\delta_{1}, \delta_{2}$ are the volume fractions and solubility parameters of the two constituent solvents, respectively. 
(a)

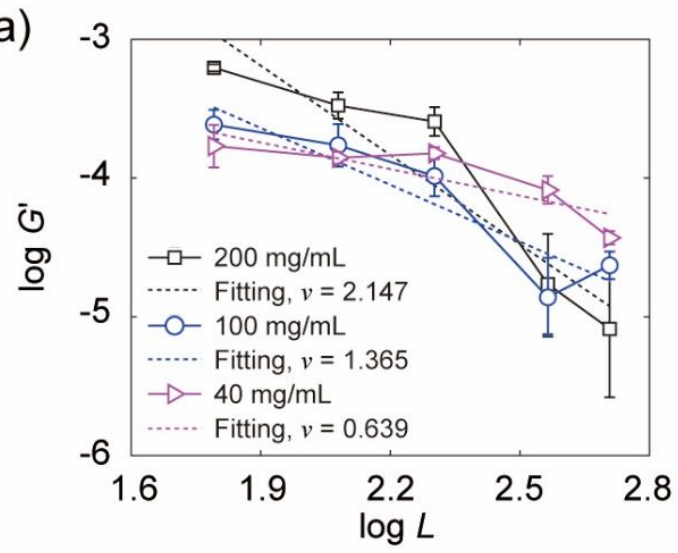

(c)

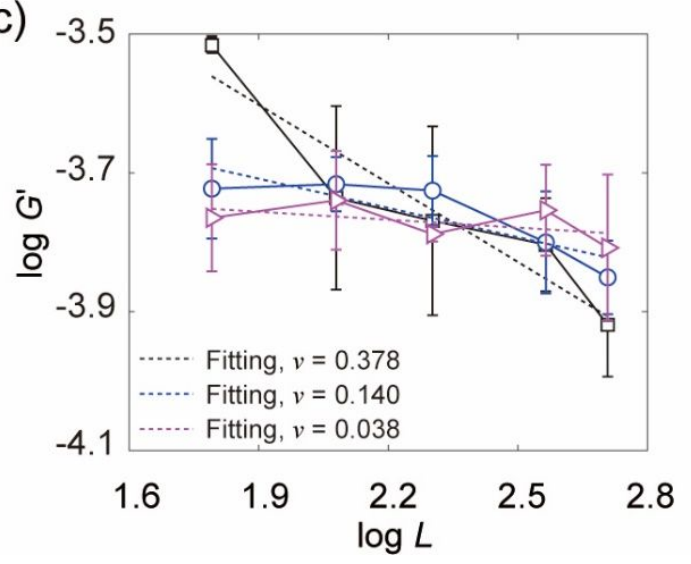

(b)

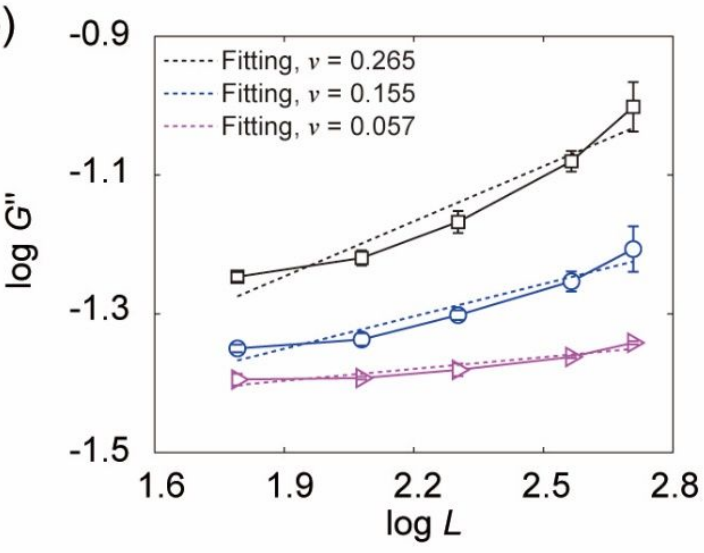

(d)

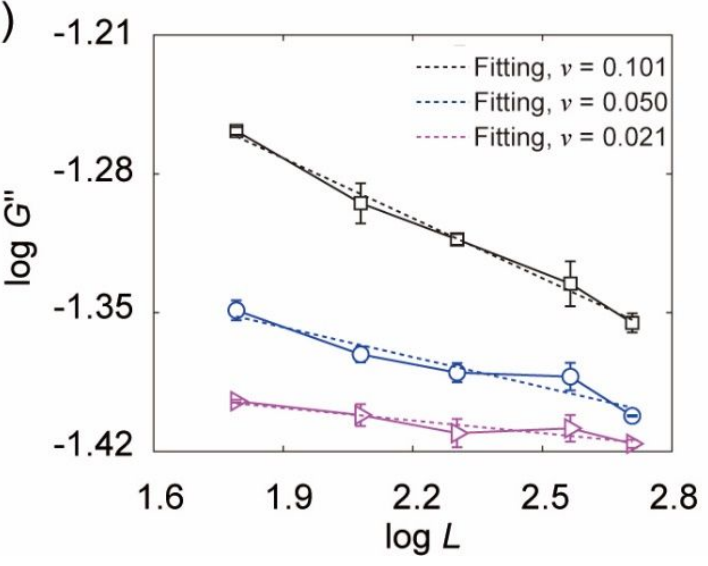

Figure S11. Storage and loss moduli $\left(G^{\prime}, G^{\prime \prime}\right)$ obtained from DPD simulations for GO solutions with (a, b) flat $\left(D=6 k_{\mathrm{B}} T\right)$ and $(\mathrm{c}, \mathrm{d})$ crumpled $\left(D=1 k_{\mathrm{B}} T\right)$ conformations. The period and amplitude of shear strain are $40 \tau$ and 0.1 , respectively. Results obtained for solutions with three concentrations are fitted for scaling relations, which are labeled as black squares $(200 \mathrm{mg} / \mathrm{mL})$, blue circles $(100 \mathrm{mg} / \mathrm{mL})$, and magenta triangles $(40 \mathrm{mg} / \mathrm{mL})$. 


\section{E. Supplemental References}

1. R. Groot, P. Warren, Dissipative particle dynamics: Bridging the gap between atomistic and mesoscopic simulation, J. Chem. Phys. 1997, 107, 4423.

2. R. Groot, K. Rabone, Mesoscopic simulation of cell membrane damage, morphology change and rupture by nonionic surfactants, Biophys. J. 2001, 81, 725.

3. D. Yllanes, S.S. Bhabesh, D. R. Nelson, M. J. Bowick, Thermal crumpling of perforated two-dimensional sheets, Nat. Commun. 2017, 8, 1381.

4. P. Poulin, R. Jalili, W. Neri, F. Nallet, T. Divoux, A. Colin, S. H. Aboutalebi, G. Wallace, C. Zakri, Superflexibility of graphene oxide, Proc. Natl. Acad. Sci. U. S. A. 2016, 113, 11088.

5. V. Symeonidis, G. E. Karniadakis and B. Caswell, Dissipative particle dynamics simulations of polymer chains: Scaling laws and shearing response compared to DNA experiments, Phys. Rev. Lett. 2005, 95, 076001.

6. I. V. Pivkin and G. E. Karniadakis, Accurate coarse-grained modeling of red blood cells, Phys. Rev. Lett. 2008, 101, 118105.

7. S. P. Koenig, N. G. Boddeti, M. L. Dunn, J. S. Bunch, Ultrastrong adhesion of graphene membranes, Nat. Nanotechnol. 2011, 6, 543.

8. B. Tang, E. Gao, Z. Xiong, B. Dang, Z. Xu, X. Wang, Transition of graphene oxide from nanomembrane to nanoscroll mediated by organic solvent in dispersion, Chem. Mater. 2018, 30, 5951 .

9. S. Plimpton, Fast parallel algorithms for short-range molecular dynamics, J. Comput. Phys. 1995, 117, 1.

10. A. Boromand, S. Jamali, J. M. Maia, Viscosity measurement techniques in dissipative particle dynamics, Comput. Phys. Commun. 2015, 196, 149.

11. H. Arkin, W. Janke, Gyration tensor based analysis of the shapes of polymer chains in an attractive spherical cage, J. Chem. Phys. 2013, 138, 054904. 
12. A. Shrake, J. A. Rupley, Environment and exposure to solvent of protein atoms. Lysozyme and insulin, J. Mol. Biol. 1973, 79, 351.

13. W. Humphrey, A. Dalke, K. Schulten, VMD: Visual molecular dynamics, J. Mol. Graph. 1996, 14, 33.

14. J. Fraaije, J. van Male, P. Becherer, R. Serral Gracia, Calculation of diffusion coefficients through coarse-grained simulations using the automated-fragmentationparametrization method and the recovery of Wilke-Chang statistical correlation, J. Chem. Theory Comput. 2018, 14, 479.

15. A. Loman, I. Gregor, C. Stutz, M. Mund, J. Enderlein, Measuring rotational diffusion of macromolecules by fluorescence correlation spectroscopy, Photochem. Photobiol. Sci. 2010, 9, 627.

16. C. Macosko, Rheology: Principles, Measurements, and Applications, Wiley-VCH: New York, United States, 1994.

17. H. Geng, B. Yao, J. Zhou, K. Liu, G. Bai, W. Li, Y. Song, G. Shi, M. Doi, J. Wang, Size fractionation of graphene oxide nanosheets via controlled directional freezing, $J$. Am. Chem. Soc. 2017, 139, 12517.

18. J. Ma, P. Wang, L. Dong, Y. Ruan, H. Lu, Highly conductive, mechanically strong graphene monolith assembled by three-dimensional printing of large graphene oxide, $J$. Colloid Interface. Sci. 2019, 534, 12.

19. C. Hansen, Three dimensional solubility parameter - Key to paint component affinities: I. Solvents plasticizers, polymer, and resins, J. Paint Technol. 1967, 39, 505. 20. A. Barton, Hanbooks of Solubility Parameters and Other Cohesion Parameters, CRC Press: Boca Raton, Florida, 1986. 